\title{
Development and administration of a screening tool for cardiovascular risk assessment in community pharmacy
}

\section{Dónal Óg O’Donovan}

School of Pharmacy, UCC

\section{Introduction}

Cardiovascular disease (CVD) was the leading cause of death in 2005, causing 17.5 million deaths globally. In Ireland, 2006 figures indicate that CVD was responsible for $43 \%$ of all deaths, and $49 \%$ of deaths in people aged less than 65 years. It is predicted to remain the largest cause of death for the foreseeable future. It has been estimated that at least $25 \%$ of CVD patients have sudden death or non-fatal myocardial infarction without prior symptoms. It is important to try to develop a method for screening these asymptomatic people. If people at high risk of CVD can be identified early then they can be treated to reduce their risk. This will in turn reduce their incidences of cardiovascular instances (heart attack, stroke, etc.) and their burden on the health service. Screening tools are used to identify those people at high risk of developing CVD. These screening tools take a number of inputs such as patient's age, gender, or blood pressure, and use these to calculate the patient's risk of CVD. Many screening tools already exist to assess CVD risk. These tools have names such as Framingham, QRISK or ASSIGN but they all perform the same task; using a patient's characteristics to calculate their risk of CVD. The drawback of these screening tools is that they all involve invasive blood tests, e.g. lipid profiling, to check a patient's cholesterol. The requirement of blood tests means that an accurate calculation of CVD risk cannot be done quickly.

\section{Aim}

The aim of this study is to pilot a novel paper-based questionnaire, relying on patient self-reporting of data in community pharmacies, that can be used to assess risk of CVD.

\section{Methodology}

Ethical approval for this project has been granted by the local ethics committee. Two hundred and ninety two participants have already been enrolled in this project. Each one of these participants has completed an extensive questionnaire to record their lifestyle and dietary habits. These questions form a database of self-reported information for each 
person. Each participant has also undergone a physical examination to check biometric information such as their blood pressure, waist circumference and cholesterol. Their risk of CVD will be calculated using established screening tools (Framingham, QRISK, ASSIGN mentioned above). Neural network software will be used to establish which self-reported questions can be used to accurately predict risk of CVD. When a range of inputs are known and the final result is also known, neural network software is used to establish which inputs affect the final result and to what extent. In this case the neural network software will identify what lifestyle and dietary information affects a person's risk of CVD in an Irish setting. These questions will then be used to form a questionnaire that will be used in community pharmacies. An expert panel of consultant cardiologists and community pharmacists will undergo a process of Delphi validation to fully validate the questionnaire. Delphi validation is a process whereby established experts in a field reach a consensus. In this case the cardiologists and community pharmacists will examine the data from the neural network and agree on the questions that should be included in the final screening tool. The final screening tool produced would be quick to apply and would consist of self-reported answers that would not require blood tests and would be quick and easy for patients to use.

\section{Work to date}

We have completed data collection for each of the 292 participants. All data has also been entered into a database ready for analysis. A pilot study has also been conducted in community pharmacies. A questionnaire was developed using European Society of Cardiology (ESC) guidelines. This questionnaire used only self-reported data. Each question was assigned a weighting by the researchers. Upon completion of the questionnaire the weightings for each risk factor were added to give a score related to their CVD risk; low, moderate or high. The questionnaire was administered in six community pharmacies in the greater Cork region.

\section{Conclusion}

The pilot study demonstrated that it is acceptable to patients to screen for CVD in community pharmacies. The questionnaire that will be developed will be fully validated and will accurately screen for risk of CVD. It will be easily to understand and quick to complete. It will be able to identify people at high risk of CVD who are asymptomatic and direct them to treatment before they suffer a cardiovascular event and become a burden on the health service.

I would like to thank my supervisors Dr. Laura Sahm and Dr. Stephen Byrne, all participants in the study so far and also my Clinical Practice postgraduate colleagues. 